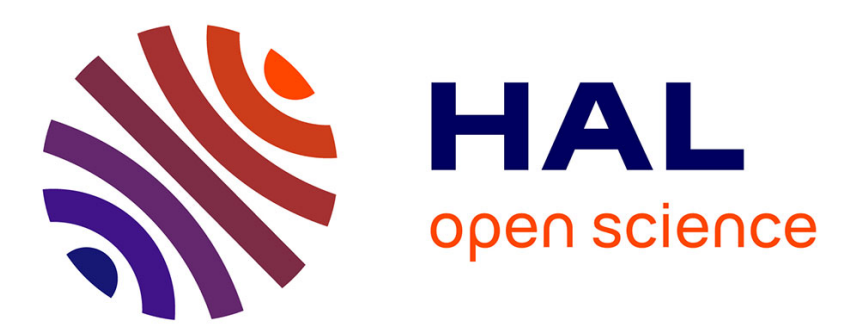

\title{
Photoswitchable Fluorescent Crystals Obtained by the Photoreversible Coassembly of a Nucleobase and an Azobenzene Intercalator
}

Li Zhou, Pascal Retailleau, Mathieu Morel, Sergii Rudiuk, Damien Baigl

\section{- To cite this version:}

Li Zhou, Pascal Retailleau, Mathieu Morel, Sergii Rudiuk, Damien Baigl. Photoswitchable Fluorescent Crystals Obtained by the Photoreversible Coassembly of a Nucleobase and an Azobenzene Intercalator. Journal of the American Chemical Society, 2019, 141 (23), pp.9321-9329. 10.1021/jacs.9b02836 . hal02363425

\section{HAL Id: hal-02363425 \\ https://hal.science/hal-02363425}

Submitted on 18 Nov 2019

HAL is a multi-disciplinary open access archive for the deposit and dissemination of scientific research documents, whether they are published or not. The documents may come from teaching and research institutions in France or abroad, or from public or private research centers.
L'archive ouverte pluridisciplinaire HAL, est destinée au dépôt et à la diffusion de documents scientifiques de niveau recherche, publiés ou non, émanant des établissements d'enseignement et de recherche français ou étrangers, des laboratoires publics ou privés. 


\title{
Photoswitchable fluorescent crystals obtained by the photoreversible co-assembly of a nucleobase and an azobenzene intercalator
}

\author{
Li Zhou, ${ }^{1}$ Pascal Retailleau, ${ }^{2}$ Mathieu Morel, ${ }^{1}$ Sergii Rudiuk, ${ }^{1}$ Damien Baigl ${ }^{1 *}$ \\ ${ }^{1}$ PASTEUR, Department of chemistry, Ecole normale supérieure, PSL University, Sorbonne \\ Université, CNRS, 75005 Paris, France \\ ${ }^{2}$ Institut de Chimie des Substances Naturelles, CNRS UPR 2301, Université Paris-Sud, \\ Université Paris-Saclay, 1 av. de la Terrasse, 91198, Gif-sur-Yvette, France.
}

*Correspondence to: damien.baigl@ens.fr.

\begin{abstract}
Self-assembled nucleobases, such as G-quartets or quadruplexes, have numerous applications but light-responsive structures are limited to small, non-crystalline motifs. In addition, the assembly of the widely exploited azobenzene photochromic compounds can produce fluorescent crystals of extended dimensions but at the prize of sacrificing their photoswitchability. Here we overcome inherent limitations of self-assembly with a new concept of supramolecular co-assembly leading in fine to materials with unprecedented properties. We show that the co-assembly of guanosine monophosphate (GMP) with an azobenzene-containing DNA intercalator produce supramolecular crystals arranged through a combination of $\pi-\pi$, electrostatic and hydrogen bond interactions. The resulting crystals are $100 \mu \mathrm{m}$ long, $\mathrm{pH}$-sensitive, fluorescent and can be photoreversibly disassembled/reassembled upon UV/blue irradiation. This allows us to perform operations such as dynamic photocontrol of a single crystal growth, light-gated permeability in membrane-like materials and photoswitchable fluorescence. We believe this concept critically expands the breadth of multifunctional materials attainable by self-assembly.
\end{abstract}




\section{Introduction}

Systems with light-switchable functions are appealing for fields ranging from biology to physics and material science. ${ }^{1-3}$ Azobenzene is one of the most attractive photochromes used in such systems because of its robust, rapid and reversible $\mathrm{E} / \mathrm{Z}$ isomerization that leads to large changes in molecular geometry and polarity. ${ }^{4}$ Applications of azobenzene derivatives in holographic memory storage, optoelectronic devices, optofluidics, molecular machines, responsive materials, energy conversion and drug design have been widely explored by taking advantage of their robust photoisomerization characteristics. ${ }^{5-11}$ However, this remarkable photoisomerization ability tends to hinder photochromic azobenzene derivatives from exhibiting fluorescence properties. By imparting azobenzene with steric rigidity, bright fluorescence can be achieved but this is usually done at the price of sacrificing the photoisomerization capability. ${ }^{12,13}$ Realizing a system displaying both azobenzene fluorescence and photoisomerization capability, an interesting combination for instance to optically modulate fluorescence, is thus a desirable challenge that has been achieved in a few rare cases. The typical strategy has consisted in controlling with light the aggregation behavior of azobenzene derivatives which, by exploiting the fluorescence enhancement effect upon aggregation, led to a variety of light-dependent aggregating systems with optically tunable emission characteristics. ${ }^{14-19}$ These materials were however usually morphologically ill-defined and poorly structured. Conversely, well-defined azobenzene crystals with bright fluorescence properties were obtained but did not display any photoswitching characteristics $^{20-22}$. Finally light-responsive organized assembly of azobenzenes were reported but the fluorescence properties were either not documented ${ }^{23}$ or modestly affected by light. ${ }^{24}$ Note that most of the above-mentioned examples were based on the assembly of hydrophobic or amphiphilic compounds in organic solvents. To our knowledge, highlyorganized azobenzene-based supramolecular structures, such as crystals, with both lightresponsive organization and on/off photoswitchable fluorescence has never been realized. Here we report the first light-responsive azobenzene crystals with photoswitchable fluorescence in water. These crystals were obtained following a new strategy consisting in the co-assembly of azobenzene-containing DNA intercalators and nucleobases as non-covalently interacting building bricks. By exploiting the natural self-arrangement characteristics of nucleobases, we found that this new way of organizing azobenzenes allowed us to obtain crystals with aggregation-induced fluorescence emission while maintaining a strong and reversible response to light stimulation. 
Among nucleobases, guanine represents a particularly interesting component for scaffolding supramolecular organizations. Due to the presence of both self-complementary donor and acceptor sites for hydrogen bonds and aromatic rings favouring $\pi-\pi$ stacking, it can self-assemble into multiple hydrogen-bonding structure like G-quartets or quadruplexes, ${ }^{25}$ showing potential in biosensors, material science and organic electronics. ${ }^{26-28}$ Efforts have also been made to expand the dynamics of the structure, which resulted in a variety of stimuli-responsive systems, the majority of them being controlled by solution composition ( $\mathrm{pH}$, ionic composition). Conversely, external control by a light stimulus has been achieved in much fewer cases and only with G-quartet structures by covalent modification or noncovalent $\pi-\pi$ stacking strategies to incorporate a photosensitive moiety. ${ }^{29-38}$ However, all of these light-sensitive structures have consisted of a few repeating units, and neither $\mu \mathrm{m}$ sized nor crystalline materials have been constructed. Here we describe for the first time light-responsive, highly organized, supramolecular architectures containing guanosine monophosphate (GMP) with a needle-like geometry and a length of several hundreds of micrometers. Both structure and light-responsiveness resulted from the co-assembly of GMP with a hydrophilic, azobenzene-containing DNA intercalator, called AzoDiGua. ${ }^{39}$ This process relied on a network of hydrogen bonds and $\pi-\pi$ stacking involving both components, resulting in a structure that is different from the G-quartet-based architecture that was reported for most GMP-based systems. In this paper, we describe the protocol for coassembling GMP and AzoDiGua, determine the structure of the resulting crystals, characterize their reversible assembling character in response to $\mathrm{pH}$ or light stimulation, and analyze their fluorescence properties. We finally exploit the light-responsiveness of these new assemblies to demonstrate a combination of unique properties, such as photoreversible assembly, optical growth control, light-gated permeability and photoswitchable fluorescence.

\section{Preparation of the supramolecular hybrid crystals}

Figure 1 shows the concept of our co-assembly method to generate hybrid crystals with both fluorescence and light-responsiveness. It consisted in the co-assembly of the GMP nucleobase with a photochrome, here an azobenzene-containing symmetric divalent guanidinium compound (AzoDiGua) having photosensitive DNA intercalating properties ${ }^{39}$ (Fig. 1A). GMP (5 mM) and AzoDiGua (0.2 mM) were mixed in acetic acid buffer $(10 \mathrm{mM}$ in water, $\mathrm{pH}=3.8$ ) at room temperature, heated to $90{ }^{\circ} \mathrm{C}$ prior to slowly cooling down to $4{ }^{\circ} \mathrm{C}$ at a speed of $1{ }^{\circ} \mathrm{C} / \mathrm{min}$ (Fig. 1B). After assembly, the suspension was brought back at room temperature (RT) and all experiments were then performed at RT. This process resulted 
in phase separation occurring only in the presence of both GMP and AzoDiGua (Fig. 1C). Optical, electron and atomic force microscopy observations of the dense phase revealed the presence of well-organized needle-like architectures with an average length and width of 101 $\pm 46 \mu \mathrm{m}$ and $1.9 \pm 0.7 \mu \mathrm{m}$ respectively (Figs. 1D, 1E, S1). Elementary analysis showed that this solid material was hexahydrated with a stoichiometry AzoDiGua:GMP of 1:2 (Table S1), corresponding to electrostatic neutralization of AzoDiGua by 2 GMP moieties in their monoanionic form $\mathrm{H}(\mathrm{GMP})^{-}$(Fig. S2). Increasing $\mathrm{pH}$ of the assembling buffer resulted in a progressive deprotonation of $\mathrm{H}_{(\mathrm{GMP})^{-}}$(Fig. S2) and led to assembled materials with reduced sizes and irregular shapes (Fig. S3). Meanwhile, sample prepared at pH 3.8 but with inosine monophosphate (IMP) or adenosine monophosphate (AMP) instead of GMP remained homogenous (Fig. S4). Compared with GMP, IMP lacks the 2-amino group that is typically engaged in one of the three H-bonds of the GC DNA base pair. Similarly, AMP provides one less hydrogen bond formation site compared with GMP. All these observations suggest that both multiple noncovalent hydrogen bonds and electrostatic interactions were involved in the successful co-assembly of GMP and AzoDiGua into extended and highly-ordered supramolecular structures.

\section{X-ray structural analysis}

We acquired X-ray diffraction data on a single crystal to gain better insight into the assembled structure and its stabilizing interactions (Fig. 2, Table S2). Interestingly, although GMP bases were not covalently bound, the relative organization of AzoDiGua and GMP was found to be reminiscent to a DNA intercalation motif (Figs. 2A, 2B), emphasizing the interest of using a photosensitive intercalator as one of the two building bricks of the supramolecular structures. The analysis showed that the azobenzene moieties in the crystal were in a twisted configuration with a $\mathrm{C}-\mathrm{C}-\mathrm{N}=\mathrm{N}$ dihedral angle of $17.9^{\circ}$ between the benzene rings and the diazo group, with the two GMP molecules arranged in a nearly parallel manner with respect to the closest benzene plane (Fig. 2B). The distance between the centroids of the AzoDiGua benzene rings and the guanine planes was $\sim 3.5 \AA$, which was within the $\pi-\pi$ interaction distance. ${ }^{40}$ The arrangement of the molecules also allowed the formation of a network of hydrogen bonds with neighbouring units to stabilize the structure (Figs. 2C, 2D, Table S3). The analysis revealed that a pair of guanine bases formed two $\mathrm{NH}_{2} \cdots \mathrm{N} 7$ hydrogen bonds $\left(2.09 \AA, 174.5^{\circ}\right)$, which explains the observed crucial role of the 2-amino group for the ordered structure formation. P-O $\cdots \mathrm{H}-\mathrm{O}$ hydrogen bonds $\left(1.76 \AA, 159.5^{\circ}\right)$ were also found between the phosphate groups of adjacent GMPs (Figure S5), which explains the importance of 


\section{pH-responsiveness of the crystals}

We analysed how the produced self-assembled crystals could be reversibly affected by the application of different stimuli targeting the non-covalent interactions involved in their structure. First, increasing the $\mathrm{pH}$ of a solution containing crystals to $\mathrm{pH}=8.5$ resulted in their rapid dissolution (Fig. 3A), due to the deprotonation of GMP and subsequent ruptures of the related hydrogen bonds. Interestingly, when the same solution was brought back to $\mathrm{pH}=4$, the sample underwent phase separation again. The re-assembled material displayed needle-like geometries of similar size and shape than the initial crystals (Fig. 3B), demonstrating that the co-assembled AzoDiGua-GMP supramolecular structure was reversibly addressable through a straightforward $\mathrm{pH}$ stimulus.

\section{Photoreversible crystal formation/dissolution}

We fixed the $\mathrm{pH}$ of the sample suspension $(\mathrm{pH}=3.8)$ and studied the effect of light on the co-assembled crystals (Fig. 4). Irradiation by UV light $(365 \mathrm{~nm})$ at RT resulted in the total dissolution of the crystals in a few minutes (Fig. 4A, Movie S1), while subsequent blue irradiation $(440 \mathrm{~nm})$ of the same solution resulted in the reformation of needle-like crystals (Fig. 4A, Movie S2). A close up analysis of the UV-induced dissolution process showed that it started by a bending of the structure prior to breaking and disappearance (Fig. 4B). A representative plot of the length of a single crystal vs time showed a two-step dissolution kinetics composed of a first slow process followed by a much faster one (Figure 4C, Movie S3). When green irradiation $(535 \mathrm{~nm}$ ) where AzoDiGua did not absorb, was used instead of $\mathrm{UV}$, neither the dissolution process nor any morphology transformation of the microstructure was detected. We thus conclude that the disassembling process comes from the photoisomerization of AzoDiGua from trans to cis isomer upon UV irradiation. ${ }^{39}$ The UVinduced bending of the crystal at the early stage of irradiation was attributed to anisotropic 
exposure to the UV stimulus leading to uneven trans-cis AzoDiGua photoisomerization features at the surface of the crystals. This speculation was supported by observing the recovery of a straight needle by applying blue irradiation (30 s) after a very short UV pulse $(0.5$ s), i.e., as soon as the crystal started to bend (Fig. S6). After repeating the process twice, a defect was observed on the crystal surface, indicating a preferential location on the crystal surface for the dissolution to start. To know whether the formation of cis-AzoDiGua upon UV irradiation was occurring only at the crystal surface or also in its core, we irradiated a crystal suspension by a moderate UV irradiation (4 min) so that the photosensitive crystals were only partially dissolved. The suspension was centrifuged immediately after UV irradiation and we compared by absorption spectroscopy the composition of the supernatant to that of the pellet re-dissolved in water (Fig. S7). Clearly, the pellet, and thus the remaining crystal structures after UV were composed of trans-AzoDiGua while cis-AzoDiGua was detected in the supernatant only, i.e., in the surrounding medium of the irradiated crystals. We can thus propose the following UV-induced crystal dissolution process. UV irradiation induced the trans-cis isomerization of AzoDiGua molecules present on the surface of the crystals, which disrupted the local $\pi-\pi$ and hydrogen bond interaction network. The cisAzoDiGua molecules disengaged from the surface and diffused into the bulk as a result of their hydrophilicity. This led to the exposure of a fresh crystal surface to the light and the disassembly process continued in a cascade-like manner until the full dissolution of the crystal. This suggested diffusion-limited mechanism explains why the full crystal dissolution required significantly more time than that for AzoDiGua to reach the photostationary state in solution under UV irradiation. ${ }^{39}$ Blue irradiation after such UV-induced dissolution was shown to trigger a re-crystallization process (Fig. 4A, Movie S2). The re-assembled material displayed needle-like structures similar to the ones before UV irradiation, but with a length typically $30 \%$ longer (Fig. 4E). Figure 4B bottom depicts the track of a representative single structure, which elongated from both ends with increased time, with an estimated initial growth speed of around $0.4 \mu \mathrm{m} \cdot \mathrm{s}^{-1}$ (Fig. 4D, Movie S3). Since AzoDiGua was shown to be rapidly converted into a trans-rich photostationary state upon blue irradiation, ${ }^{39}$ we propose the following mechanism of blue-induced recrystallization. After UV-irradiation, the photostationary cis-rich state contained a small fraction of trans-AzoDiGua molecules which were probably engaged in trans-AzoDiGua-GMP clusters that were too small to be detected by optical microscopy. Blue irradiation strongly enriched the solution into trans-AzoDiGua molecules that progressively either co-assembled on preformed structures or nucleated new ones. The presence of trans-AzoDiGua-GMP clusters after UV irradiation might thus act as 
nuclei and explain the formation, after blue irradiation, of crystals that were larger and in a smaller number than before the UV irradiation step (Fig. S8). All these results show that the photoisomerization properties of AzoDiGua were successfully preserved in the selfassembled structures to generate $100 \mu \mathrm{m}$-long azobenzene-GMP crystals with photoreversible assembly capability.

\section{Dynamic photocontrol of a single crystal growth}

In materials science, controlling the kinetics of material growth until desired dimensions, once the parameters like temperature and concentration are set, is a desirable challenge. We thus explored how the dimension of a single needle-shaped crystal could be controlled in a remote and dynamic way by light (Figs 4F, 4G, Movie S4). To this end, we locally irradiated a crystal suspension using a focused UV beam to fully dissolve the solid material in the irradiated area. Then a much smaller focused laser spot of blue light was applied in front of a selected needle and switched on and off in a dynamic manner. Figs. 4F and $4 \mathrm{G}$ show that the growth of the needle was strictly correlated with the application of the light stimulus. The needle grew at almost constant speed when the light was on while the growth was momentarily stopped as long as the light was kept off. This unique feature allowed us to dynamically control the growth of the targeted single crystal by light with precise spatiotemporal resolution.

\section{Light-gated permeable material}

We then explored how the photoreversible assembly/disassembly properties demonstrated on individual crystals could be exploited to control the permeability of extended materials composed of a large number of photoresponsive crystals. We first prepared a thin layer of crystals and found that the successive application of focused UV and blue light allowed us to dynamically open and close a single pore at the lit area (Figs. 5A, 5B, Movie S5). The UV-induced pore formation was also successful with a thicker layer $(\sim 100$ $\mu \mathrm{m})$ but required prolonged irradiation time. Interestingly, the size and shape of the lightinduced pore reproduced well the spot dimensions, allowing us to create a hole in the material with an arbitrary, user-defined shape (Figs. 5C, 5D, Movie S6). We then embedded the photoresponsive crystals inside a thin layer of agarose gel to form a $0.9 \mathrm{~mm}$ thick membrane that we placed, in a sandwich configuration, between two aqueous buffer compartments (Fig. $5 \mathrm{E})$. The upper compartment was initially loaded with Rhodamine B dye (RhB) and we measured the absorbance of the lower compartment solution 20 min after loading $\mathrm{RhD}$ in the 
upper chamber. The absorbance spectrum displayed a peak around the absorption maximum of $\mathrm{RhB}(554 \mathrm{~nm})$ showing that the dye diffused through the membrane. Interestingly, the absorbance was approximately 6 times higher when the membrane was exposed to UV, demonstrating an enhanced permeability of the membrane material upon UV exposure. These simple proof-of-concept demonstrations show that the photoreversible assembly character of the crystals can be easily implemented to design and build materials with user-controlled and light-gated permeability.

\section{Photoswitchable fluorescence}

We also found that the supramolecular AzoDiGua-GMP crystals were fluorescent upon blue excitation (Ex/Em 488/493-575 nm) allowing us to observe them by fluorescence microscopy (Fig. 6A). The crystal material displayed a maximal emission at around $600 \mathrm{~nm}$ and a wide excitation range from $350 \mathrm{~nm}$ to $500 \mathrm{~nm}$ (Fig. 6B). Notably, under the same excitation conditions, the fluorescence emission of the hybrid crystal solution was 58 times stronger than AzoDiGua alone (Fig. S9). Apart from a much lower intensity, the emission spectrum of AzoDiGua alone displayed similar features as that of the crystal. This shows that the fluorescence properties mainly emerged from the specific supramolecular organization of AozDiGua within the co-assembled crystal. Compared to AzoDiGua alone, the absorption of AzoDiGua in the crystal displayed a red-shift attributed to the $\pi-\pi$ and hydrogen bond interaction (Figure S10). The absorption band that associated with the $\pi-\pi^{*}\left(\mathrm{~S}_{2} \leftarrow \mathrm{S}_{0}\right)$ transition of the azobenzene unit shifted from $357 \mathrm{~nm}$ to $370 \mathrm{~nm}$. Meanwhile, a new strong shoulder band appeared at around $470 \mathrm{~nm}$, where the forbidden $n-\pi^{*}\left(\mathrm{~S}_{1} \leftarrow \mathrm{S}_{0}\right)$ transition of the trans isomer is located. In principle, since the fluorescence from the S1 excited state in solution is extremely weak due to its short lifetime and its optically forbidden nature, it is hardly observed. We thus attribute the observed fluorescence in needle crystals to a relaxation mechanism of the S1 excited state in the co-crystalline state that is different from that of AzoDiGua alone in solution. Crystallography data showed a restricted conformation of AzoDiGua due to its arrangement with neighbouring GMPs making rotation or inversion relaxation processes upon photoexcitation not favourable. We thus propose that the suppression of the nonradiative relaxation was responsible for the greatly enhanced fluorescence emission. Similar effects have been regularly reported in azobenzene crystals but all fluorescent crystalline systems reported so far lost most of their photoisomerization ability upon crystallization. Here, the unique supramolecular nature of the co-assembled AzoDiGua-GMP crystals allowed us to maintain their photoswitching characteristics (Figs. 4- 
5). We thus exploited the combination of the photoreversible assembly with the crystallization-induced fluorescence to analyse how fluorescence could be modulated by light in our system. Interestingly, applying UV on a crystal suspension allowed us to switch off the fluorescence while subsequent blue irradiation on the same solution induced a remarkable recovery of the fluorescence (Fig. 6C).

\section{Conclusion}

We have proposed a new concept of supramolecular assembly associating a nucleobase, here GMP, with an azobenzene-containing photosensitive DNA intercalator called AzoDiGua. This strategy allowed us to obtain properties that could not be achieved by conventional assembly of the same family of compounds taken separately. For instance, we obtained the first $100 \mu \mathrm{m}$ long GMP-based crystals, while conventional photoresponsive guanine assemblies involving G-quartets or quadruplexes have been mainly limited to small non-crystalline structures of a few repeating units. Similarly, this co-assembly principle allowed us to build a new family azobenzene crystals combining for the first time fluorescence and photoswitchability. Hold by a synergic network of $\pi-\pi$, electrostatic and hydrogen bond interactions, the resulting crystals presented a combination of unique properties such as photoreversible assembly, optical shapability and photoswitchable fluorescence, and could be integrated in membrane-like materials to achieve interesting functionality such as light-gated permeability. We believe that this concept can be easily extended to other molecular bricks carrying additional functionality, thus constituting a promising framework for the facile preparation of a variety of user-defined, stimuliresponsive, and multifunctional self-assembled materials.

\section{References}

1. Russew, M.-M. \& Hecht, S. Photoswitches: From Molecules to Materials. Adv. Mater. 22, 3348-3360 (2010).

2. Brieke, C., Rohrbach, F., Gottschalk, A., Mayer, G. \& Heckel, A. Light-controlled tools. Angew. Chem. Int. Ed. 51, 8446-8476 (2012).

3. Baigl, D. Photo-actuation of liquids for light-driven microfluidics: state of the art and perspectives. Lab Chip 12, 3637-3653 (2012).

4. Fliegl, H., Köhn, A., Hättig, C. \& Ahlrichs, R. Ab Initio Calculation of the Vibrational and Electronic Spectra of trans - and cis -Azobenzene. J. Am. Chem. Soc. 125, 98219827 (2003). 
5. Kassem, S. et al. Artificial molecular motors. Chem. Soc. Rev. 46, 2592-2621 (2017).

6. Beharry, A. A. \& Woolley, G. A. Azobenzene photoswitches for biomolecules. Chem. Soc. Rev. 40, 4422-4437 (2011).

7. Matharu, A. S., Jeeva, S. \& Ramanujam, P. S. Liquid crystals for holographic optical data storage. Chem. Soc. Rev. 36, 1868 (2007).

8. Lubbe, A. S., Szymanski, W. \& Feringa, B. L. Recent developments in reversible photoregulation of oligonucleotide structure and function. Chem. Soc. Rev. 46, 1052 1079 (2017).

9. Diguet, A. et al. Photomanipulation of a droplet by the chromocapillary effect. Angew. Chem. Int. Ed. 48, 9281-9284 (2009).

10. Estévez-Torres, A. et al. Sequence-independent and reversible photocontrol of transcription/expression systems using a photosensitive nucleic acid binder. Proc. Natl. Acad. Sci. U. S. A. 106, 12219-12223 (2009).

11. Venancio-Marques, A., Barbaud, F. \& Baigl, D. Microfluidic mixing triggered by an external LED illumination. J. Am. Chem. Soc. 135, 3218-3223 (2013).

12. Bisle, H. \& Rau, H. Fluorescence of noncyclic azo compounds with a low-lying 1(n, $\left.\pi^{*}\right)$ state. Chem. Phys. Lett. 31, 264-266 (1975).

13. Yoshino, J. et al. Intensely Fluorescent Azobenzenes: Synthesis, Crystal Structures, Effects of Substituents, and Application to Fluorescent Vital Stain. Chem. Eur. J. 16, 5026-5035 (2010).

14. Shimomura, M. \& Kunitake, T. Fluorescence and photoisomerization of azobenzenecontaining bilayer membranes. J. Am. Chem. Soc. 109, 5175-5183 (1987).

15. Tsuda, K. et al. Fluorescence from Azobenzene Functionalized Poly(propylene imine) Dendrimers in Self-Assembled Supramolecular Structures. J. Am. Chem. Soc. 122, 3445-3452 (2000).

16. Han, M. \& Hara, M. Intense Fluorescence from Light-Driven Self-Assembled Aggregates of Nonionic Azobenzene Derivative. J. Am. Chem. Soc. 127, 10951-10955 (2005).

17. Han, M. R., Hirayama, Y. \& Hara, M. Fluorescence Enhancement from SelfAssembled Aggregates: Substituent Effects on Self-Assembly of Azobenzenes. Chem. Mater. 18, 2784-2786 (2006).

18. Tsai, B.-K., Chen, C.-H., Hung, C.-H., Hsiao, V. K. S. \& Chu, C.-C. Photoswitchable fluorescence on/off behavior between cis- and trans-rich azobenzenes. J. Mater. Chem. 22, 20874 (2012).

19. Ren, H., Chen, D., Shi, Y., Yu, H. \& Fu, Z. A carboxylic azo monomer and its homopolymer: synthesis, self-organization and fluorescence behaviour in solution. Polym. Chem. 6, 270-277 (2015).

20. Jee, A.-Y., Lee, Y., Lee, M. \& Kim, M. H. Communication: Time-resolved fluorescence of highly single crystalline molecular wires of azobenzene. J. Chem. Phys. 136, 121104 (2012).

21. Han, M. et al. Multistimuli-responsive azobenzene nanofibers with aggregationinduced emission enhancement characteristics. Chem. Commun. 50, 15815-15818 (2014).

22. Han, M., Takeoka, Y. \& Seki, T. Facile morphological control of fluorescent 
nano/microstructures via self-assembly and phase separation of trigonal azobenzenes showing aggregation-induced emission enhancement in polymer matrices. J. Mater. Chem. C 3, 4093-4098 (2015).

23. Lee, S. et al. Stimulus-Responsive Azobenzene Supramolecules: Fibers, Gels, and Hollow Spheres. Langmuir 29, 5869-5877 (2013).

24. Han, M., Cho, S. J., Norikane, Y., Shimizu, M. \& Seki, T. Assembly of an Achiral Chromophore into Light-Responsive Helical Nanostructures in the Absence of Chiral Components. Chem. Eur. J. 22, 3971-3975 (2016).

25. Gellert, M., Lipsett, M. N. \& Davies, D. R. Helix formation by guanylic acid. Proc. Natl. Acad. Sci. 48, 2013-2018 (1962).

26. Davis, J. T. G-Quartets 40 Years Later: From 5'-GMP to Molecular Biology and Supramolecular Chemistry. Angew. Chem. Int. Ed. 43, 668-698 (2004).

27. Berger, O. et al. Light-emitting self-assembled peptide nucleic acids exhibit both stacking interactions and Watson-Crick base pairing. Nat. Nanotechnol. 10, 353-360 (2015).

28. Pu, F., Wu, L., Ran, X., Ren, J. \& Qu, X. G-Quartet-Based Nanostructure for Mimicking Light-Harvesting Antenna. Angew. Chem. Int. Ed. 54, 892-896 (2015).

29. Mayer, G., Kröck, L., Mikat, V., Engeser, M. \& Heckel, A. Light-Induced Formation of G-Quadruplex DNA Secondary Structures. ChemBioChem 6, 1966-1970 (2005).

30. Heckel, A. et al. An Anticoagulant with Light-Triggered Antidote Activity. Angew. Chem. Int. Ed. 45, 6748-6750 (2006).

31. Kim, Y., Phillips, J. A., Liu, H., Kang, H. \& Tan, W. Using photons to manipulate enzyme inhibition by an azobenzene-modified nucleic acid probe. Proc. Natl. Acad. Sci. 106, 6489-6494 (2009).

32. Ogasawara, S. \& Maeda, M. Reversible Photoswitching of a G-Quadruplex. Angew. Chem. Int. Ed. 48, 6671-6674 (2009).

33. Lena, S., Neviani, P., Masiero, S., Pieraccini, S. \& Spada, G. P. Triggering of Guanosine Self-Assembly by Light. Angew. Chem. Int. Ed. 49, 3657-3660 (2010).

34. Wang, X. et al. Conformational Switching of G-Quadruplex DNA by Photoregulation. Angew. Chem. Int. Ed. 49, 5305-5309 (2010).

35. Schmidt, T. L., Koeppel, M. B., Thevarpadam, J., Gonçalves, D. P. N. \& Heckel, A. A Light Trigger for DNA Nanotechnology. Small 7, 2163-2167 (2011).

36. Rivera, J. M. \& Silva-Brenes, D. A Photoresponsive Supramolecular G-Quadruplex. Org. Lett. 15, 2350-2353 (2013).

37. Thevarpadam, J. et al. Photoresponsive Formation of an Intermolecular Minimal GQuadruplex Motif. Angew. Chem. Int. Ed. 55, 2738-2742 (2016).

38. Tian, T. et al. Small-Molecule-Triggered and Light-Controlled Reversible Regulation of Enzymatic Activity. J. Am. Chem. Soc. 138, 955-961 (2016).

39. Bergen, A. et al. Photodependent Melting of Unmodified DNA Using a Photosensitive Intercalator: A New and Generic Tool for Photoreversible Assembly of DNA Nanostructures at Constant Temperature. Nano Lett. 16, 773-780 (2016).

40. Janiak, C. A critical account on $\pi-\pi$ stacking in metal complexes with aromatic nitrogen-containing ligands. J. Chem. Soc. Dalt. Trans. 3885-3896 (2000). doi:10.1039/b003010o 
Acknowledgements. This work was supported by the European Research Council (ERC) [European Community's Seventh Framework Programme (FP7/2007-2013)/ERC Grant Agreement No. 258782] and by the "PSL-Chimie" program. We thank Y. Chen for providing access to his SEM apparatus.

\section{Author Information:}

- The authors declare no competing financial interests

- Correspondence and requests for materials should be addressed to Damien Baigl (damien.baig1@ens.fr) 
A)

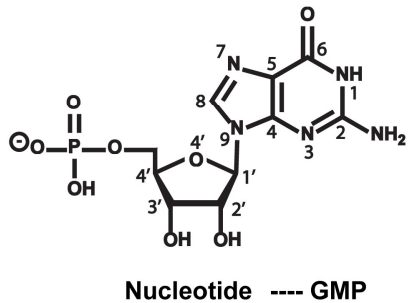

B)

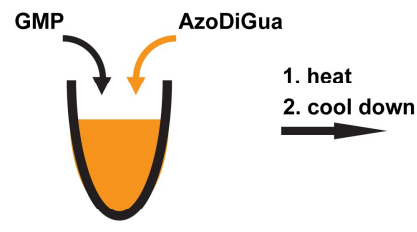

no fluorescence

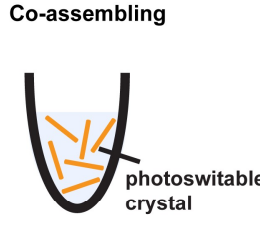

fluorescence on

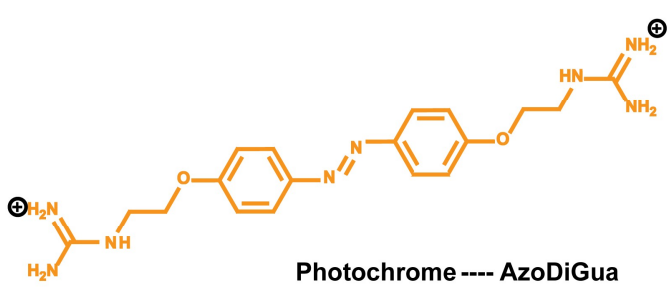

disassembling
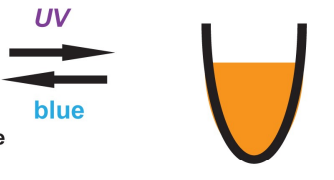

C)
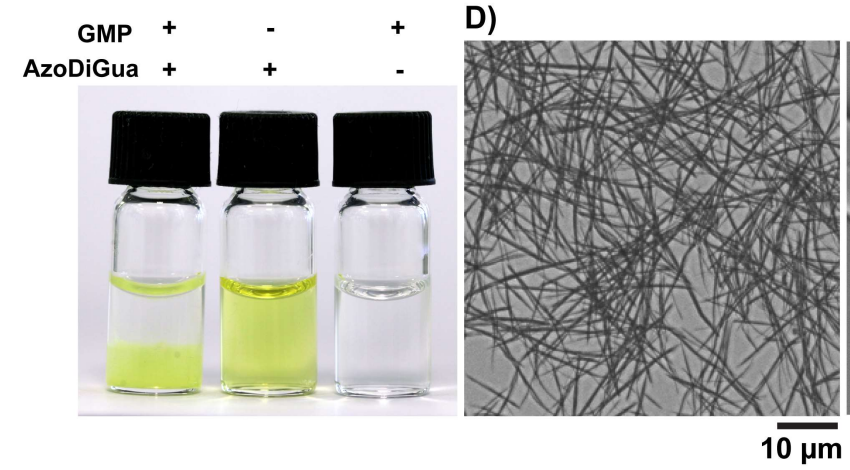

E)

fluorescence off

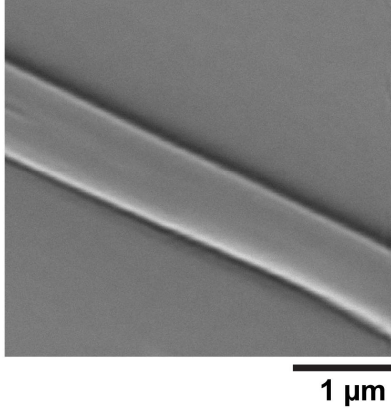

Figure 1. Building a fluorescent, photo-switchable crystal by co-assembly of a nucleobase and a photochromic intercalator. A) Molecular structures of the nucleobase, guanosine monophosphate (GMP), and the photochrome, a symmetric azobenzene diguanidinium compound (AzoDiGua), used in this study. B) GMP (5mM) and AzoDiGua $(0.2 \mathrm{mM})$ were assembled in acetic acid buffer $(10 \mathrm{mM}$ in water, $\mathrm{pH}=3.8)$, heated to $90^{\circ} \mathrm{C}$ and slowly cooled down to $4{ }^{\circ} \mathrm{C}$ to form photoswitchable fluorescent crystals. After assembly, crystals were characterized and manipulated at room temperature (RT). C) Photographs of solutions prepared by different combinations of GMP and AzoDiGua, $20 \mathrm{~min}$ at RT after the assembly process. D-E) Transmission optical (D) and scanning electron (E) microscopy images of the resulting crystals deposited on glass after drop casting and water evaporation. 


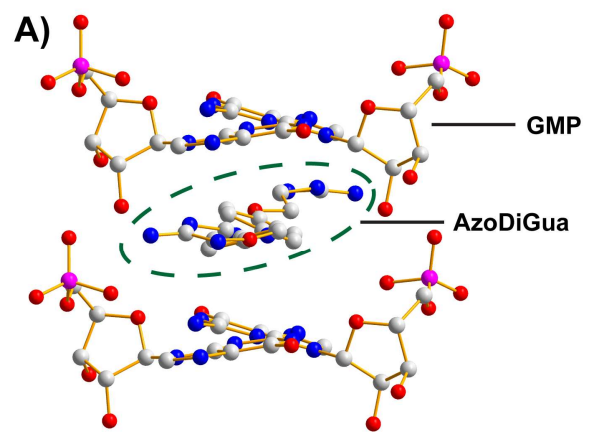

B)

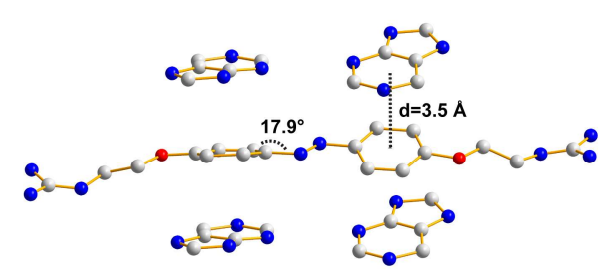

C)

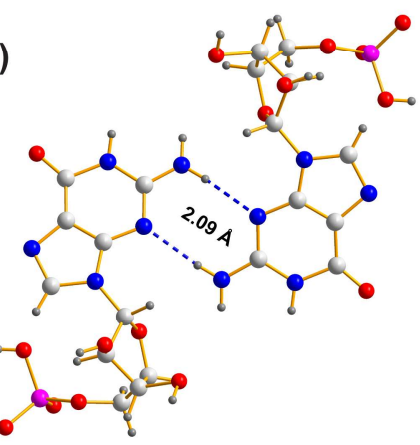

(D)

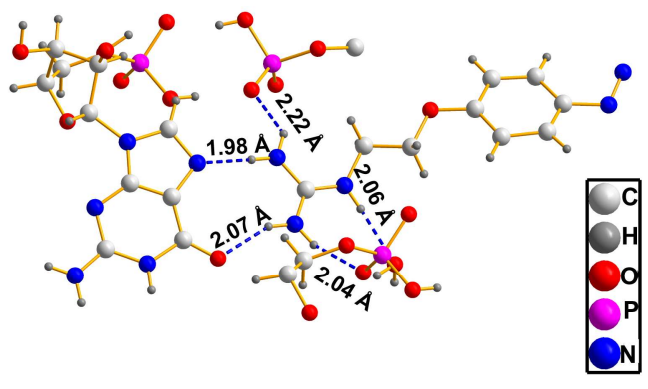

Figure 2. Single crystal X-ray crystallography reveals the structure of the GMP/AzoDiGua assemblies. A) Packing diagram of GMP/AzoDiGua crystal. B) $\pi-\pi$ interaction between AzoDiGua and GMP in the crystal. C) Intermolecular hydrogen bonds between two adjacent GMPs. D) The guanidium group of AzoDiGua forms a network of hydrogen bonds with neighbouring units. In (A) and (B), hydrogen atoms and functional groups are omitted for clarity. 


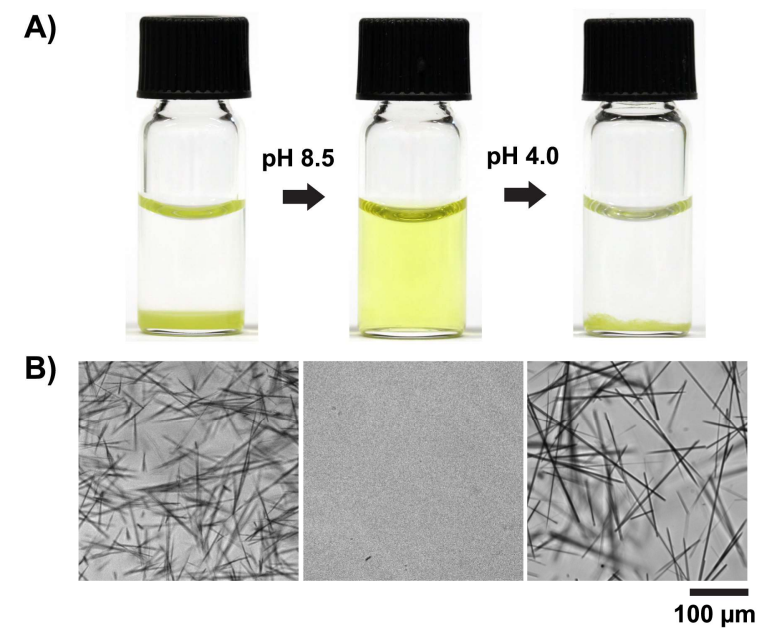

Figure 3. Hybrid crystals are pH-responsive. Photograph of the suspension (A) and in situ transmission optical microscopy images (B) before (left) and after successive mixing of $\mathrm{NaOH}$ (middle) and $\mathrm{HCl}$ (right) at $\mathrm{RT}$. The $\mathrm{pH}$ of the solution was 3.8, 8.5 and 4.0, respectively. 

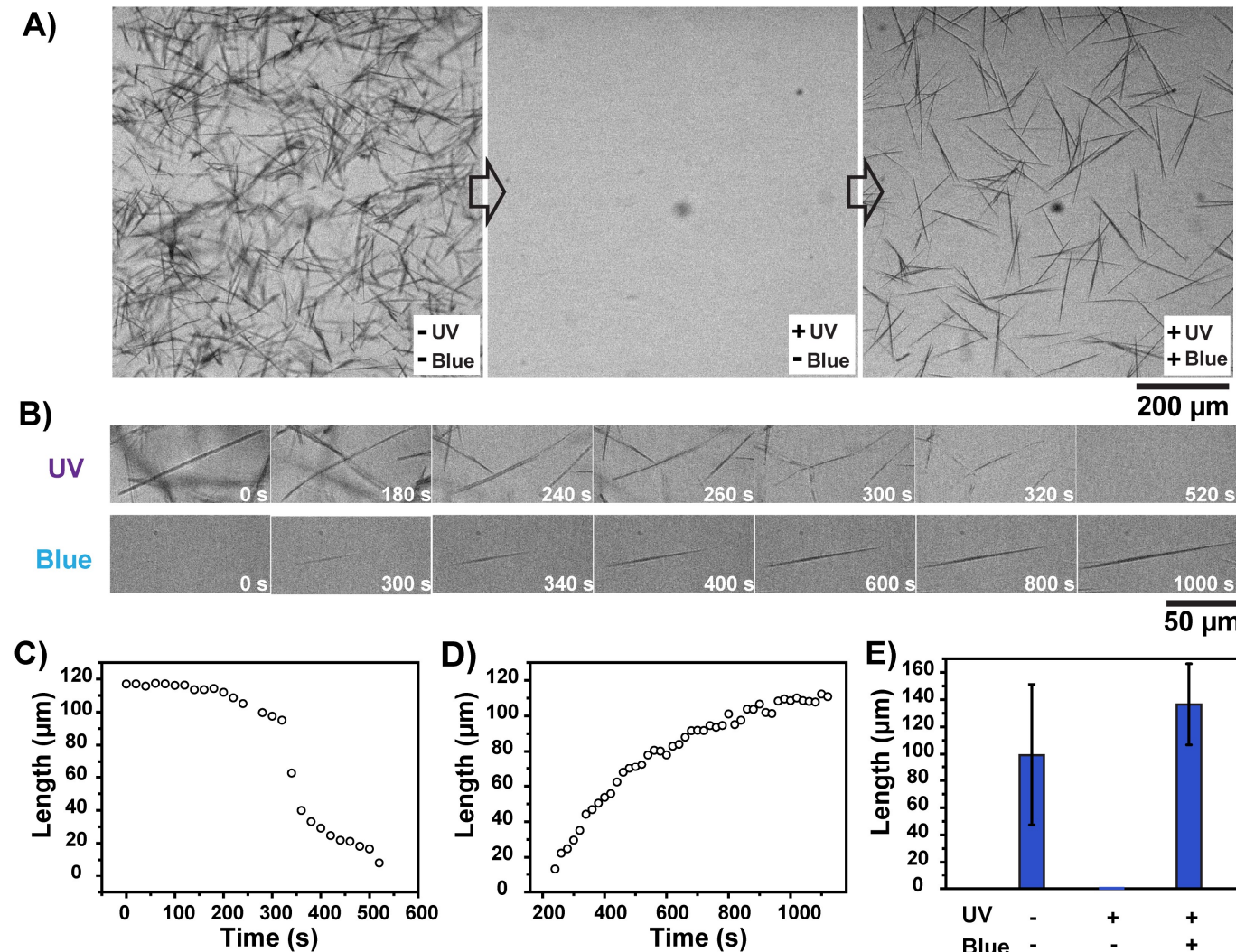

F)

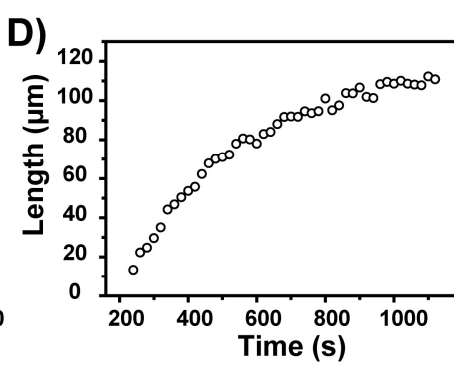

Time (s)

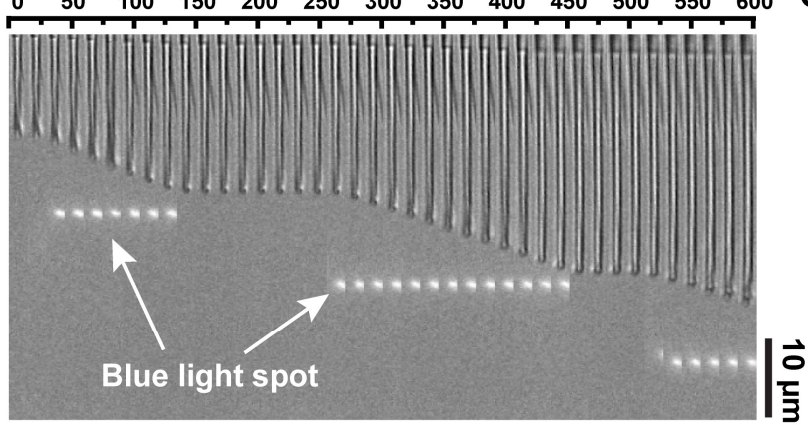

$200 \mu \mathrm{m}$
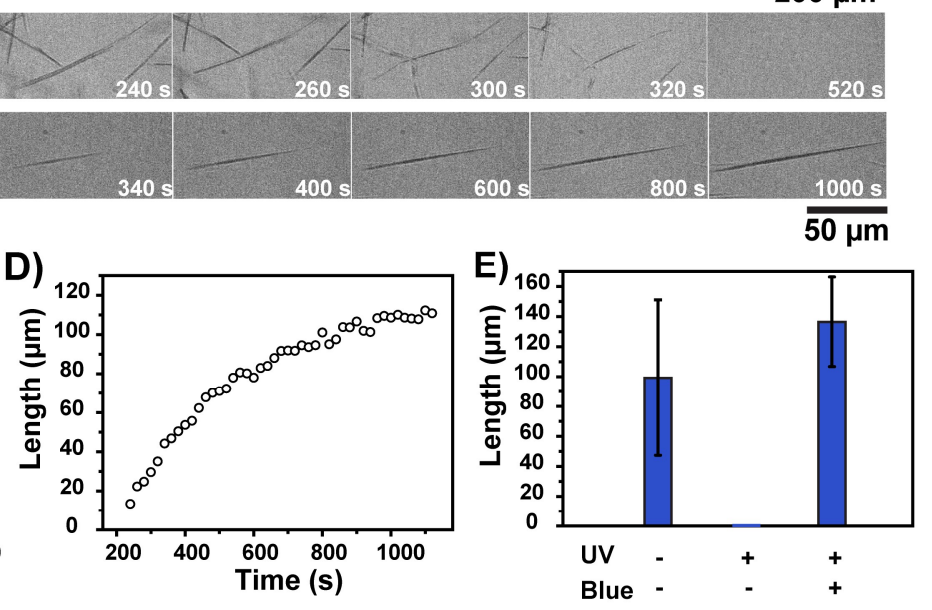

G)

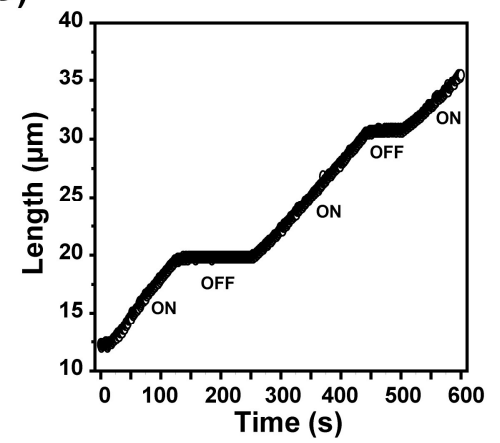

Figure 4. Reversible photocontrol of crystallization. A-E) In situ, real-time transmission optical microscopy characterization of a suspension of crystals before (-UV -Blue), and after successive irradiation by UV $\left(365 \mathrm{~nm}, 2.3 \mathrm{~mW} \cdot \mathrm{cm}^{-2}\right)$ for $10 \mathrm{~min}(+\mathrm{UV}-\mathrm{Blue}$,$) and by blue$ $\left(440 \mathrm{~nm}, 16 \mathrm{~mW} \cdot \mathrm{cm}^{-2}\right)$ for $5 \mathrm{~min}(+\mathrm{UV}+$ Blue $)$. Wide-field representative images in a given irradiated area (A). Time lapse observation and corresponding crystal length of the UVinduced dissolution (B top, C) and of the blue-induced recrystallization (B bottom, D) of a single crystal. Average crystal length (mean \pm sd on 100 crystals) for the successive irradiation conditions (E). F-G) High-magnification transmission optical microscopy observation of the recrystallization of a single crystal guided by a blue light spot (488 nm, spot size $1.6 \mu \mathrm{m}$ ) in a solution previously irradiated by a larger UV light spot (365 nm, spot size $412 \mu \mathrm{m}$ ) to locally dissolve crystals in the irradiated area. The blue spot irradiation zone 
was made visible by the fluorescence emission of fluorescein added to the buffer ( $1 \mathrm{nM})$. Kymograph of the crystal growth (F). Length of the crystal versus time (G). 


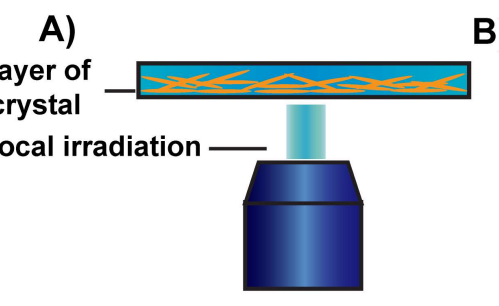

B)
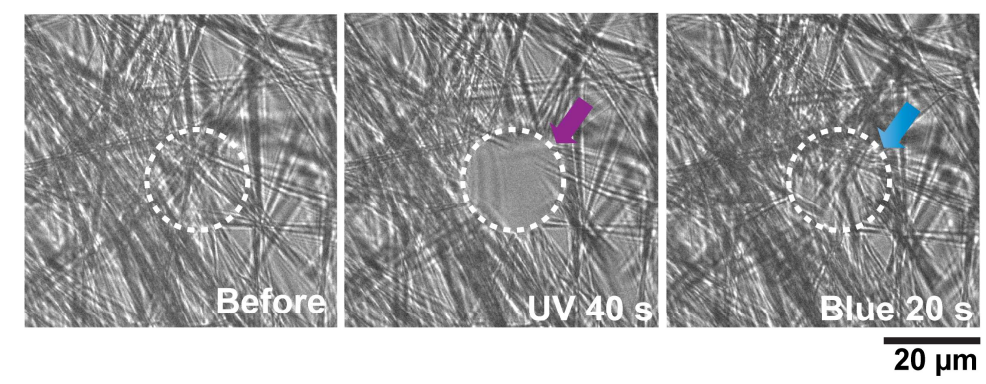

C)
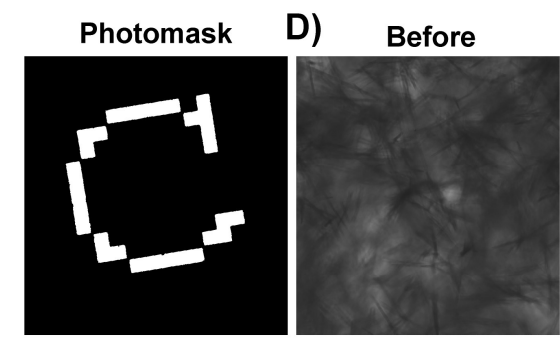

UV $10 \mathrm{~min}$

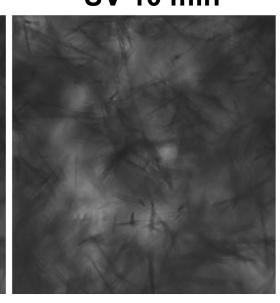

UV $17 \mathrm{~min}$
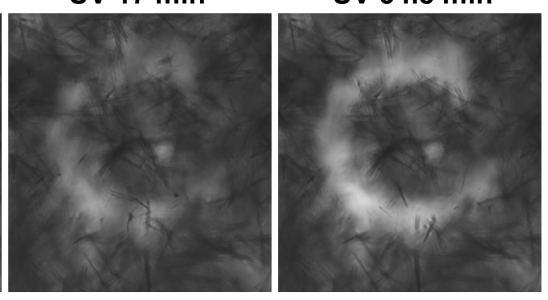

E)

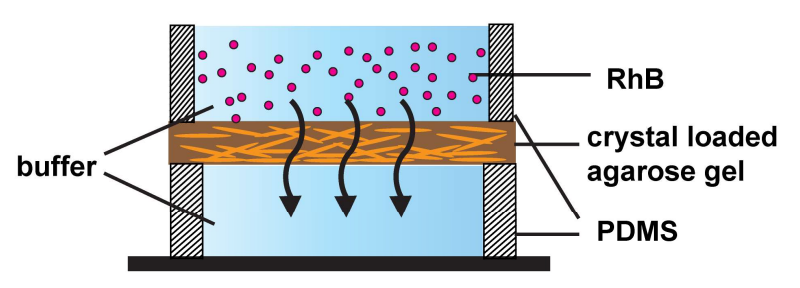

F)

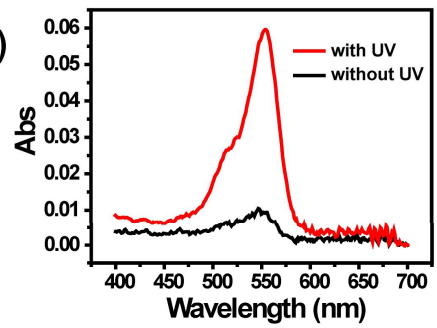

$\overline{100 \mu \mathrm{m}}$

Figure 5. Light-gated permeability in a crystal layer. A-B) A thin layer of crystals is locally irradiated with a circular spot $\left(921 \mu \mathrm{m}^{2}\right)$ inside the suspension buffer (A). In situ transmission microscopy images (B) before (left) and after successive irradiation by UV $\left(365 \mathrm{~nm}, 4 \mathrm{~mW} \cdot \mathrm{cm}^{-2}\right)$ for $40 \mathrm{~s}$ (middle) and blue $\left(440 \mathrm{~nm}, 28 \mathrm{~mW} \cdot \mathrm{cm}^{-2}\right)$ for $20 \mathrm{~s}$ (right). The dashed circle indicates the irradiated area. C-D) A thick layer (approx. $100 \mu \mathrm{m}$ ) of crystals is locally irradiated by UV through a photomask displaying the letter " $\mathrm{C}$ " (C). In situ transmission of the crystal layer a function of irradiation time (D). E-F) A $0.9 \mathrm{~mm}$ thick hybrid membrane composed of agarose and crystals is placed between two polydimethylsiloxane (PDMS) compartments. The upper ( $8 \mathrm{~mm}$ diameter) and the lower (3 $\mathrm{mm}$ diameter) compartments contain GMP $(4.6 \mathrm{mM})$ in acetic acid buffer $(10 \mathrm{mM}$ in water, $\mathrm{pH}=3.8)$ while Rhodamine $\mathrm{B}(\mathrm{RhB}, 0.1 \mathrm{mM})$ is supplemented only in the upper one $(\mathrm{E})$. Absorption of the solution in the bottom compartment 20 min after the addition of $\mathrm{RhB}$, with (red curve) or without (black curve) continuous irradiation by UV $\left(365 \mathrm{~nm}, 11 \mathrm{~mW} \cdot \mathrm{cm}^{-2}\right)(\mathrm{F})$. 

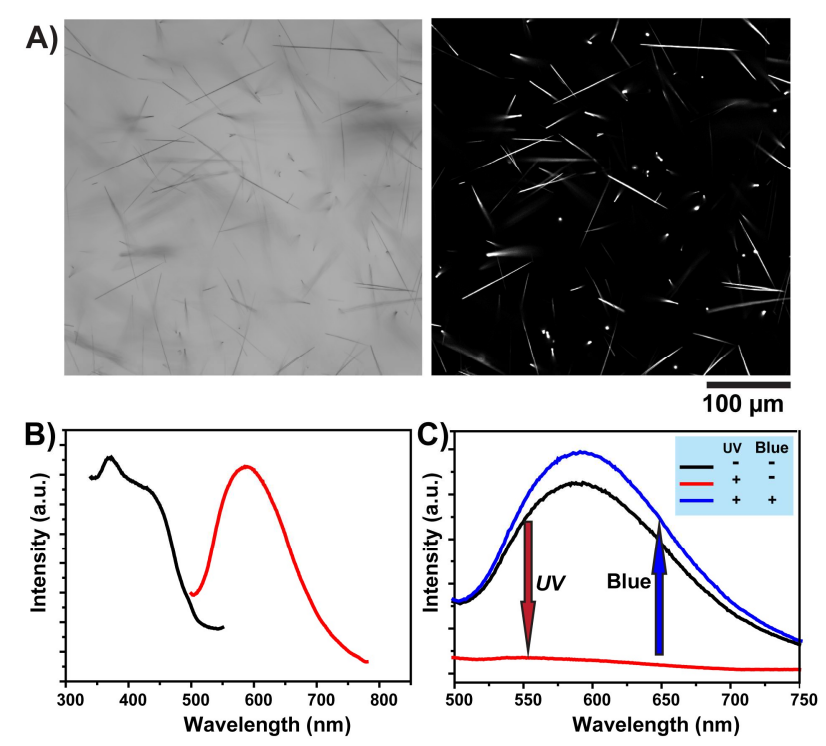

Figure 6. Photoswitchable fluorescent crystals. A) Transmission (left) and fluorescence (right, 488/493-575 nm) images of crystals in the suspension buffer. B) Fluorescence excitation (at $597 \mathrm{~nm}$ emission, black curve) and emission (at $400 \mathrm{~nm}$ excitation, red curve) spectrum of the crystal suspension. C) Fluorescence emission spectrum (excitation $410 \mathrm{~nm}$ ) of a crystal suspension (200 $\left.\mu \mathrm{L}, 0.6 \mathrm{~g} \cdot \mathrm{L}^{-1}\right)$ before (black curve) and after successive irradiation by UV (365 $\mathrm{nm}, 11 \mathrm{~mW} \cdot \mathrm{cm}^{-2}$ ) for $25 \mathrm{~min}$ (red curve) and blue (440 $\mathrm{nm}$, $16 \mathrm{~mW} \cdot \mathrm{cm}^{-2}$ ) for $10 \mathrm{~min}$ (blue curve). 